\title{
Why are some numerical concepts more successful than others? An evolutionary perspective on the history of number concepts
}

\author{
Helen De Cruz \\ Center for Logic and Philosophy of Science, Free University of Brussels, Pleinlaan 2, Brussels 1050, Belgium \\ Initial receipt 1 November 2005; final revision received 24 February 2006
}

\begin{abstract}
From the history of mathematics, it is clear that some numerical concepts are far more pervasive than others. In a densely multimodular mind, evolved cognitive abilities lie at the basis of human culture and cognition. One possible way to explain the differential spread and survival of cultural concepts based on this assumption is the epidemiology of culture. This approach explains the relative success of cultural concepts as a function of their fit with intuitions provided by conceptual modules. A wealth of recent evidence from animal, infant, and neuroimaging studies suggests that human numerical competence is rooted in an evolved number module. In this study, I adopted an epidemiological perspective to examine the cultural transmission of numerical concepts in the history of mathematics. Drawing on historical and anthropological data on number concepts, I will demonstrate that positive integers, zero, and negative numbers have divergent cultural evolutionary histories owing to a distinct relationship with the number module. These case studies provide evidence for the claim that science can be explained in terms of evolved cognitive abilities that are universal in Homo sapiens.
\end{abstract}

(C) 2006 Elsevier Inc. All rights reserved.

Keywords: Numerical cognition; Cognitive evolution; Epidemiology of culture; Cultural evolution; History of mathematics

Elements of this article were presented at the 27th Annual Meeting of the Cognitive Science Society, Stresa, Italy, July 21-24, 2005.

This research was supported by Grant OZR916BOF from the Free University of Brussels.

E-mail address: hdecruz@vub.ac.be. 


\section{Introduction}

Scientific knowledge has shown a staggering and explosive increase over the past five centuries. Mathematical knowledge has played an important role in this evolution by providing surprisingly efficient formulations to look into and organize empirical phenomena as well as by being a source of scientific creativity. A debate has emerged on the question of whether the evolution of science has depended largely on cultural and social factors or on intrinsic cognitive ones. To be sure, extrinsic factors such as the invention of printing have played an important role. However, disagreement exists on the question of to what extent innate cognitive abilities continue to play a role in science. Some scholars (e.g., McCauley, 2000) hold that science requires cognitive skills that have no basis in cognitive evolution; science can only arise in specific cultural conditions through deliberate practice set in highly institutionalized environments. Others (e.g., Brewer, Chinn, \& Samarapungavan, 2000) insist that there is a fundamental continuity between scientific and nonscientific modes of thought, as even young children prefer parsimonious and coherent explanations over ad hoc and incoherent accounts. If this latter view were true, then there is no major discontinuity between scientific and intuitive modes of reasoning and science could be explained in terms of evolved cognitive abilities that are universal in humans. One way to test these opposing views is to examine as to what extent evolved cognitive abilities have influenced the invention and spread of concepts in the history of science. In this study, I adopted this approach to examine the extent to which our evolved number sense has influenced the history of mathematics in Europe and elsewhere.

Numerical concepts, such as symbolic representations of positive integers, are the result of cultural evolution: a gradual accumulation of mathematical knowledge within different cultures. Nevertheless, recent evidence from animal and infant studies suggests that mathematical concepts are rooted in an evolved number sense that does not require cultural transmission. At present, the question of to what extent this innate number sense influences cultural transmission has not been fully explored. Geary (1995) persuasively argued that most complex mathematical knowledge can only be acquired as a result of deliberate and sustained practice that is especially designed and maintained for this purpose. This, however, does not exclude the possibility that innate numerical intuitions continue to influence the transmission of cultural mathematical concepts. My aim in this article was to explore the role of our innate number sense in the transmission of cultural numerical concepts. To this end, I adopted an epidemiological approach (Sperber, 1996) to explain the differential success of positive integers, zero, and negative numbers as a function of their relationship with innate cognitive abilities.

The article begins with an overview of the relationship between conceptual modularity and cultural transmission. It takes the perspective that the mind consists of many specialized conceptual modules as a starting point and examines how human culture can be explained in terms of conceptual modularity. Next, I review experimental evidence from cognitive ethology, developmental psychology, and neuroscience which indicates that humans and other vertebrates share a highly specialized neural system for detecting numerosities in the environment. I then examine the cultural transmission of three types of numerical concepts- 
positive integers, zero, and negative numbers - through an epidemiological lens. Each of these concepts displays a different type of cultural transmission owing to a distinct relationship with the number sense.

\section{Conceptual modularity and culture}

\subsection{Conceptual modularity}

Animals' brains enable them to behave adaptively even in situations that they have not encountered. How does the brain create adaptive responses based on sensory input? Cosmides and Tooby (1994) contended that the brain consists of many specialized systems - conceptual modules - that have their own specific way of dealing with a given subset of computational problems; in other words, they are domain specific. Because problems encountered by humans and by other animals are often mutually incompatible, natural selection has crafted a dedicated solution for each recurrent evolutionary problem. For instance, our species has a rich social life that requires specialized social cognition. Social relationships come in many forms: we make friends, avoid fights, and have to assess whether or not we can trust someone. The rules we observe for making friends are not the same as those we use when detecting cheaters; thus, these two competencies are probably governed by separate modules. We can therefore predict that our cognitive architecture will be densely multimodular (Tooby \& Cosmides, 1995). Taking this a step further, Sperber (1994) and Gallistel (1995) adopted the view that the brains of humans and other animals are entirely made of modules: there are, in other words, no domain-general cognitive systems.

How can mass modularity be compatible with the great diversity of human cultural abilities? Most elements of human culture are too novel and too variable to be the specific output of any module. Opera, oil refineries, priesthood celibacy, and kayaks to hunt seals from are all recent cultural inventions. Sperber (1994) drew a useful distinction between proper and actual domains of modules. The proper domain is the reason a module exists; it defines the function of the module and is part of its evolutionary history (e.g., the proper domain of a face-recognition module is human faces). The actual domain is the set of stimuli to which the module responds, whether it belongs to its proper domain or not. For the face-recognition module, its actual domain consists not only of real human faces but also of photographs, drawings, and sculptures of faces. The slight mismatch between the proper and actual domains is systematically exploited in human culture (Sperber \& Hirschfeld, 2004). Because humans acquire most of their information through culture, a large part of domain-specific knowledge is cultural. Modules are likely to respond to cultural stimuli that have a strong concurrence with their proper domains. Masks, for example, are part of the artistic traditions of many cultures because they tap into the domain of the face-recognition module by exaggerating some of the prominent characteristics of faces. Culturally transmitted domain-specific concepts can flourish even if they have no basis in the real world, such as imaginary creatures and, as I shall argue, numerical concepts. 


\subsection{Epidemiological approaches to culture}

Cultural representations have differential transmission success: some occur much more frequently than unconstrained variation would predict. Religious concepts such as weeping statues or spirits are universal, although no one has actually witnessed them. Although ecological relevance may account for the success of some cultural ideas (e.g., hunting techniques), it does not plausibly account for the salience of seemingly less useful ideas (e.g., religious concepts). This has led several anthropologists to suggest that cultural transmission is biased by evolved psychological predispositions. Cultural traits that build upon preexisting intuitions from conceptual modules will enjoy a much greater success than cultural traits with a poor correspondence to conceptual modules because they are easier to reconstruct.

Intuitive concepts are found widely across cultures. They cluster around a small set of recurrent features, such as folk biology, folk psychology, and folk physics. These bodies of knowledge develop in young children without explicit teaching. The "theory of mind," for example, matures similarly across widely differing cultures (Callaghan et al., 2005): children typically pass a standardized false belief test between the ages of 4 and 5 years. Cultural elaborations upon these core intuitions are easy to learn and are typically part of normal and spontaneous cognitive development during infancy and childhood. Next to intuitive concepts, psychological evidence indicates that minimally counterintuitive cultural traits, such as religious concepts, may also have a transmission advantage. They have a limited number of features that violate intuitive assumptions (Boyer, 2001). Ghosts, for instance, violate naive physics because they can walk through walls or disappear at will. On the other hand, they behave in accordance with naive psychology by having beliefs, desires, and social intercourse. Thus, minimally counterintuitive concepts provide a cognitive optimum because they activate an evolved inference system (theory of mind) that supports many inductive inferences and can therefore be represented without allocating too many cognitive resources while at the same time recruiting a lot of attention by violating a limited number of assumptions. Lastly, nonintuitive concepts are relatively rare across cultures. Such concepts typically violate a large number of intuitions, making it difficult to represent them coherently or to generate predictions and inferences. Indeed, psychological evidence reveals that people distort maximally counterintuitive ideas to fit them more into intuitive expectations. Christian and Hindu college students (Barrett, 1998; Barrett \& Keil, 1996), for example, do not intuitively think about their respective gods as their theologies require. They have difficulties representing them as omniscient, omnipresent beings and distort stories about them to fit intuitive expectations that they have about normal people. Unsurprisingly, the transmission of nonintuitive concepts can only be sustained in highly institutionalized contexts, where long training and external storage of information overcome the cognitive limitations of the individual (McCauley, 2000).

To conclude, it can be argued that there are not one but several tracks of cultural inheritance because cultural concepts activate different sets of conceptual modules (Boyer, 2000). Because each module imposes its own organization on a distinct type of knowledge, it is crucial to examine how these work. To explain patterns of cultural transmission of mathematical concepts, we need to examine how they interact with intuitions provided by the 
number module. In the next section, I briefly review the experimental literature that supports the existence of a conceptual number module.

\section{Number as the proper domain of a conceptual module}

Numbers are abstract representations that seem far removed from elementary sensory data. They appear to imply knowledge of language: arithmetic, counting, and more complex computations involving number rely on linguistic tools. It seems, therefore, unlikely that numbers could constitute the proper domain of one or more dedicated modules. On the other hand, number is a basic property of the environment. From an evolutionary perspective, stable properties of the environment that yield potentially useful information can exert selective pressures on nervous systems in animals. Therefore, we can expect that modules that infer gravitational pull, color, or numerosities occur in many species. Recognizing numerosities provides animals with a mechanism to reduce complicated forms of input (objects or events in time and space) to simple numerical relationships.

\subsection{Numerical competence in nonhuman, animals, human infants, and adults}

Current experimental evidence from diverse disciplines suggest that numbers are more than a cultural invention and that human numerical competence has its roots in cognitive evolution. Numerical competence is present in human infants, prior to their schooling or even language acquisition. Similar capacities have been found in animals even in the absence of training. Furthermore, functional neuroimaging studies strongly suggest that number processing rests on a distinct neural circuitry. Scientists from various disciplines have, based on this, argued that humans and other vertebrates share an evolved number sense (in the terminology of Dehaene, 1997), a specialized neural mechanism for detecting numerosities in the environment.

The evolutionary roots of numerical abilities are perhaps best illustrated by animals that spontaneously use numerical cues when making adaptive decisions. For example, lionesses decide whether or not to attack an intruding group based on a comparison of the number of unfamiliar roaring individuals they hear and the number of members of their pride present (McComb, Packer, \& Pusey, 1994). Animals also rely on numerical cues to guide their foraging decisions: when presented with two patches of food items, both rhesus monkeys (Hauser, Carey, \& Hauser, 2000) and red-backed salamanders (Uller, Jaeger, Guidry, \& Martin, 2003) go for the larger quantity.

Human infants share this capacity to reason about number. Newborns of just a few days old discriminate between sets of two and three items but fail to see the difference between four and six items (Antell \& Keating, 1983). Combined with similar findings from animal studies, some authors (e.g., Uller, Hauser, \& Carey, 2001) proposed that animals and human infants are able to represent numerosities up to 3 or 4 exactly but fail to represent higher numerosities. However, experiments suggest that infants can represent larger numerosities approximately provided that the ratio difference is large enough: 6-month olds dishabituate 
when the number of dots on a display changes from 8 to 16 when other variables such as total surface area have been controlled for (Xu \& Spelke, 2000). Nonetheless, only humans are able to represent numbers larger than 3 or 4 precisely; even chimpanzees after long periods of training fail to do so (Hauser, 2005).

Adults rely on numerical concepts similar to those of infants and other animals when they are prevented from counting. If adults are asked to press a key a specific number of times, while being prevented from subvocal counting, the mean number of key presses increases in proportion to the target number (Cordes, Gelman, \& Gallistel, 2001). Adults' accuracy and speed in numerical performance get worse as the absolute size of the numbers increases (size effect) and as the distance between them gets smaller (distance effect) (e.g., a comparison between 7 and 8 typically takes a longer response time than that between 3 and 8 ; Moyer \& Landauer, 1967). In sum, animals as well as human infants and adults who do not count fail to make exact representations of all but the smallest quantities. From an evolutionary perspective, this seeming fuzziness may actually prove to be more efficient than a linear, exact representation of a number. This is because a plausible candidate for the proper domain of the number module may be calculating return rates when making foraging decisions (Gallistel, 1990). After all, the difference between 1 apple and 2 apples is more ecologically significant than that between 50 and 100 apples.

\subsection{The neural architecture underlying numerical competence}

The neural representation of number has been studied through various neuroimaging techniques. Models that invoke conceptual modularity at the neural level suggest that there are neural circuits specialized in domain-specific, semantic-level processing of information, such as plants and animals (Caramazza \& Mahon, 2003). Several neuroimaging studies (e.g., Dehaene, Spelke, Pinel, Stanescu, \& Tsivkin, 1999) indicate that the horizontal banks of the intraparietal sulci (HIPS) represent the neural correlate of a conceptual module that deals only with numerical information. Their activation can be reliably dissociated from nonnumerical tasks that require a similar level of attention, working memory, and spatial cognition (Simon, Mangin, Cohen, Le Bihan, \& Dehaene, 2002). For example, the functional magnetic resonance imaging study by Eger, Sterzer, Russ, Giraud, and Kleinschmidt (2003) found that the presentation of an Arabic digit (say 3) activates the HIPS, whereas that of a similarly presented letter (say $a$ ) does not. Even when presented in an auditory format, the word two elicits activation in the HIPS, whereas the word red does not. Thus, the HIPS are activated in the most elementary and cross-modal recognition of numerosities; their activation cannot be explained as a byproduct of the task demands of numerical operations.

How can individual neurons recognize numerosities? In a set of single-cell studies, Nieder and Miller (2003) presented rhesus monkeys with pairs of slides with dots that varied in size, shape, or numerosity. They found individual neurons that responded only to changes in number and remained insensitive to changes in shape or size. Each number neuron shows a peak activity to a specific quantity and becomes progressively less active as magnitude increases. For example, a neuron optimally activated by two is less responsive to one or three and even less so to more items. Because neurons are only coarsely tuned, close numerosities 
will activate similar populations of neurons. Smaller numerosities yield narrower tuning curves, thus making them easier to distinguish; larger numerosities yield broader tuning curves, thus making their discrimination fuzzier.

\section{The epidemiology of numerical concepts}

Numerical concepts vary widely between cultures, yet some are more widespread than others. One way to explain these patterns of cultural transmission is by adopting the epidemiological approach. To date, the relationships between evolved cognitive abilities and culture have been mainly examined for religion and supernatural concepts (e.g., Boyer \& Ramble, 2001), folk biology (e.g., Atran, 1998), and folk sociology (e.g., Sperber \& Hirschfeld, 2004). Numerical concepts also constitute a viable candidate. We can expect that some cultural numerical concepts may enjoy a greater success of transmission because they correspond closely to the intuitions provided by the number sense. On the other hand, numerical concepts that minimally violate intuitive expectations can thrive because they are surprising while not posing too much computational demand. Finally, numerical concepts that maximally violate numerical intuitions can only be transmitted in highly institutionalized contexts because external storage of information and highly trained staff are required for their survival. In the following sections, I show that positive integers, zero, and negative numbers have a different transmission rooted in their varying relationships with our evolved number module. For each case, I examine experimental psychological evidence and historical patterns of cultural diffusion.

\subsection{The positive integers}

Some developmental psychologists (e.g., Wynn, 1998) believe that positive integers $\{1,2$, $3, \ldots\}$ constitute the psychological foundation from which all other numerical concepts arise. The apparent ease of individual acquisition (poverty of the stimulus) and ease of cultural diffusion seem to indicate that positive integers could constitute the proper domain of a conceptual module. However, as we have seen, the number sense makes only approximate representations that grow increasingly imprecise as numerosities increase. Hence, positive integers must be human cultural inventions. The magnitudes that animals as well as human infants and adults who do not count represent are more properly conceptualized as reals than as integers. Reals (e.g., $\sqrt{ } 2, \pi)$ are uncountable: they cannot be put in a one-to-one correspondence with natural numbers. We can conceptualize reals as being on a continuous scale, whereas the positive integers constitute a discrete set (Gallistel \& Gelman, 2000). Because we do not intuitively reason about numerosities in terms of positive integers, any model that explains the success and salience of positive integers in various cultures has to explain why these are apparently intuitively appealing and culturally widespread and why reals are not. To date, no language that completely lacks number words has been recorded. Nevertheless, there is considerable variation in their occurrence across cultures: some cultures have elaborate positional number word systems, whereas others have a very limited number word vocabulary. 
Recent investigations in two indigenous South American hunter-gatherer societies showed that some cultures do not possess true discrete numerical concepts. The Pirahã (Gordon, 2004) have only words for one, two, and many — sometimes using their word for one for small collections of items as well. In controlled experiments, they can discriminate between very small sets such as two and three but their performance for three and four is at chance level. The Mundurukú (Pica, Lemer, Izard, \& Dehaene, 2004) are also unfamiliar with counting. They too use number words (up to five) in an approximate rather than precise fashion. For instance, when 5 dots were presented, the subjects responded five in only $28 \%$ of the trials. However, they are able to discriminate between sets of 20 and 80 dots, not unlike human infants and animals.

These studies indicate that the mere presence of number words does not suffice to promote an exact representation of numerosities. More crucial perhaps is that both Amazonian cultures lack a counting routine (i.e., putting objects in a one-to-one correspondence). This results in a conceptual transformation in which the continuous magnitude scale provided by the number module is supplemented by a number line consisting of discrete elements (the positive integers). This connection between one-to-one correspondence and number theory was already recognized by the mathematician Cantor (1845-1918). Without the principle of oneto-one correspondence, a true understanding of positive integers as discrete entities does not emerge, plainly because the number-sensitive neurons' representations are too fuzzy to enable discrete representations. Conversely, humans are able to make exact representations of magnitude without number words. Patients with extreme aphasia have been demonstrated to show a remarkably conserved ability to make exact multiple digit calculations (Varley, Klessinger, Romanowski, \& Siegal, 2005), suggesting that language is not a prerequisite for exact calculation. Moreover, positive integers can be represented by nonlinguistic symbols as well (e.g., a shepherd may keep track of the size of his flock by putting tallies into a one-toone correspondence with his sheep as they move between shelter and pasture; Ifrah, 1985). Thus, not language per se but counting by sequential tagging is the key ingredient to a successful understanding of positive integers.

The activity of counting involves a relationship between three sets: countable items, counting symbols, and mental magnitudes (Fig. 1A). The set of items to be counted varies from one count to another. To count, one establishes a one-to-one correspondence between each item and a conventionally defined list of symbols that have a fixed ordinality (e.g., the number words one, two, and three; sequential tagging). The final item tagged determines the last tag from the counting sequence, which in turn denotes the cardinality of the set. This highest number word is mapped onto a corresponding mental magnitude. Through cultural learning, a synaptic connection gets established between these tags and the corresponding mental magnitude. Thus, the population of neurons in the HIPS that fires preferentially around three will respond to any symbolic representation for this magnitude. In this way, it becomes possible to establish a discrete, exact representation of magnitude by mapping an ordered set of symbols onto mental magnitudes. As shown in Fig. 1A, Arabic numerals that denote positive integers $\{1,2,3, \ldots\}$ are directly translated onto a continuous and approximate magnitude scale. Temple and Posner (1998) demonstrated that a number comparison task with Arabic numerals (say 5 and 3) yields an almost identical pattern of brain activation as one that involves arrays of dots (say five dots and three dots). Thus, the 

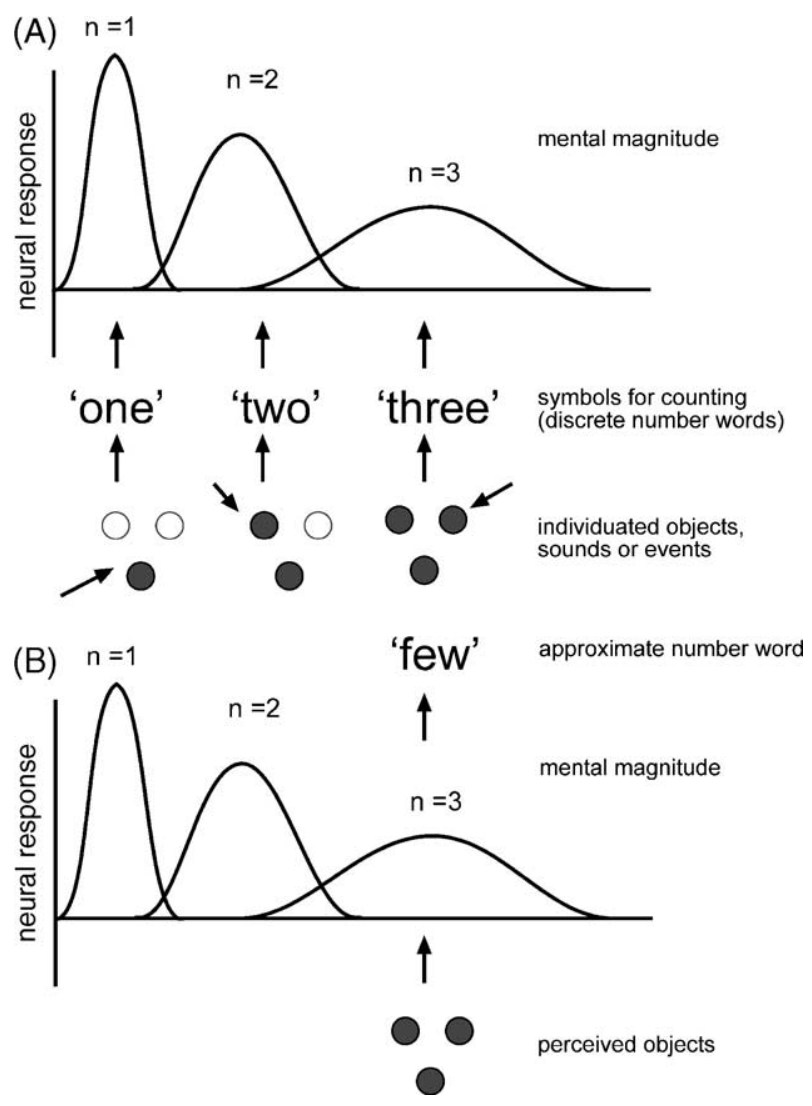

Fig. 1. The linguistic representation of numbers in a counting sequence (A) and approximately (B).

brain immediately translates a positive integer into a mental representation of its quantity. In contrast, the mental representation of approximate number words follows a different neural trajectory (Fig. 1B). Here, cardinality is established directly, without sequential tagging. This approximate representation is subsequently converted into a linguistic expression, the approximate number word (e.g., few, a couple, about a hundred). Western adults also use this mechanism when accurate answers are difficult to come by (e.g., "There are about 300 students in my Introduction to Philosophy class.").

If counting is not an evolved ability, then why has it emerged so frequently? Late Paleolithic artifacts (ordered series of notches on bone and antler) indicate that positive integers were represented as early as 20,000 years ago, long before writing was invented (d'Errico, 1998). Understanding positive integers and the counting routine seemingly develops spontaneously and with little formal teaching in Western and Papua children (Saxe, 1981). Before they can successfully count, preschoolers have the intuition that the application of a positive integer changes when the numerosity of items changes (Sarnecka \& Gelman, 2004). Taken together, this evidence lends support to the suggestion that the counting procedure itself may constitute a precursor to the positive integer system. 
To ensure that we count each item in a set exactly once, we shift our attention to each of them in a systematic, ordered way. This can be achieved by gesturing or gaze direction. Indeed, under experimental conditions, children have more difficulties keeping track when they are prevented from pointing (Alibali \& DiRusso, 1999). Gesturing helps lighten the cognitive demands of counting because it enables us to individuate objects in space more easily (Goldin-Meadow \& Wagner, 2005). The neural correlates of gesturing and visual attention lie very close to the HIPS (Simon et al., 2002). The formation of new synaptic connections may be easier between two anatomically adjacent areas. Culture may key in on this architectural property of the human brain by creating synaptic connections between them through learning how to count. Similarly, establishing a one-to-one correspondence between an ordered list of numerical symbols and mental magnitudes often exploits the structure of the brain. Many cultures use body parts (often fingers) as number symbols, which explains the wide occurrence of base-5, base-10, and base20 positional systems (Butterworth, 1999). Neuropsychological evidence indicates that the identification of body parts is a conceptual module, the body schema, whose impairment leads to a disability to identify one's own body parts. It is an ideal candidate for a list of symbols with fixed ordinality (number symbols) because it also represents body parts in an ordered fashion: the comparison of body parts is prone to a distance effect similar to that in number comparison (e.g., to determine that the nose is lower than the eyes takes a longer response time than judging that the knees are lower than the eyes; Le Clec'H et al., 2000). Thus, one always has a list with fixed order at hand, so to speak. The neural correlates of the body schema lie adjacent to the HIPS, in the left intraparietal lobule. A temporary disruption in this area (through rTMS) results in a marked increase in reaction time when subjects complete a number comparison task, suggesting that finger counting continues to play an important part in adult numerical cognition (Sandrini, Rossini, \& Miniussi, 2004).

In brief, although positive integers are intuitive, they are not universal. Although they do not constitute the proper domain of the number module, they can become part of its actual domain through the cultural scaffolding provided by the counting procedure. This counting procedure is a cultural construction that is derived from the modules for number, language, attention direction, and body-part identification. As a result, positive integers are anchored in more than one conceptual module, as is the case for many complex cultural concepts such as religion, art, and mathematics.

\subsection{Zero}

The concept of zero as a numerical value has created opportunities in Western mathematics that could never have been realized without it, extending to almost all domains of mathematics. It might therefore seem strange that zero is not widespread across cultures and their mathematical systems. From an evolutionary perspective, however, this is not surprising. Number-sensitive neurons discharge preferentially at different numerosities. Zero obviously means an absence of neural activation in the number-sensitive areas of the brain. Because these areas are informationally encapsulated, they cannot reflect on their own lack of activation. Experiments (e.g., Wynn \& Chiang, 1998) lend empirical support to this hypothesis. Eight-month olds were confronted with either "magical" or expected events. In 
the $1-1=1$ or 0 condition, they saw one object on a stage, a screen then occluded it, and a hand visibly removed it. Once the screen was removed, the infants saw either the expected result (no object) or a magical appearance in which the removed object was still there. Intriguingly, subjects showed no surprise to this last result $(1-1=1)$ ! In contrast, earlier studies (e.g., Wynn, 1992) showed that infants are able to predict that $2-1=1$ and not 2 . These findings suggest that the number module is not capable of representing zero, leaving infants unable to form the expectation that no object will be seen.

How then did zero come into existence? Zero as a byproduct of a positional system that needed a symbolic representation for an empty placeholder emerged several times independently (e.g., Babylon, Maya, Inca, and India). However, the use of an empty placeholder does not automatically lead to a true numerical concept for zero, as aptly illustrated by Mayan mathematics. The Maya had a concept of zero as a result of their base- 20 positional system. Unfortunately, cosmological and religious considerations (the significance of their solar calendar) created an anomaly at the third position, which was set at $20 \times 18=360$ (instead of the straightforward $20 \times 20=400$ ) because 360 is the number of days in their solar year. This anomaly deprived the Mayan zero from its potential calculative properties (Ifrah, 1985).

Zero as a numerical value has its roots in classical Indian mathematics. The oldest Indian mathematical concepts can be found in the Vedas, a collection of religious texts dating between 1500 and $500 \mathrm{BC}$, and the accompanying Vedangas, which contains sutras, rules that were of vital importance to the performance of ritual offerings. Large public offerings required altars constructed from complex geometric figures. If incorrectly constructed, the offerings would be of no value. Considering the effort, means, and time invested in each public offering, its success was crucial. This increased the cultural importance of mathematical concepts such as geometry, arithmetic, and (a precursor to) algebra. An additional incentive was provided by the fascination for large numerosities, salient in classical Indian poetry. It originated from stylistic considerations: because large numerosities impressed readers, poets felt compelled to use ever-increasing magnitudes to emphasize the age, size, or distance of any event, building, or other thing they described. This led to the introduction of words that could express powers of 10. Combined with words that denote single digits up to 9, this enabled the elegant and parsimonious formulation of very large numbers. Parsimony was important for Vedic texts that were (and still are) learnt by heart. This eventually resulted in the invention of a positional system in which a symbol for the empty placeholder became essential. However, it was only in Jain mathematics that zero became a fully fledged mathematical concept (Joseph, 1990). As a reaction to Brahmin orthodoxy, heterodox Jains no longer practiced complex offerings. Severed from its religious origins, mathematics became a discipline studied for its own sake. The Jains had a fascination for mathematical concepts such as infinity, positing several types of infinite sets centuries before Cantor. Words for nothingness such as shunya meant more to them than absence or void and implied receptiveness. Because of their cosmological ideas of time and space, emptiness could be conceptualized as a thing in its own right instead of merely being an empty placeholder. This led Hindu mathematicians such as Brahmagupta (598-668) to wonder how numerical operations with zero could be performed (e.g., whether or not division by 0 is possible). This mature mathematical concept of zero subsequently spread to China and 
Southeast Asia as well as to the Islamic world, from which it diffused to the West. The first mention of zero outside India is found in 7th-century Nestorian and Syrian Orthodox writings. Its spread was facilitated by their interest in Indian astronomy and the search for efficient methods to calculate an accurate date for Easter. By the beginning of the 11th century, various sources illustrate that zero had spread throughout the Arab empire both by scholars and nonscholars. The oldest mention of zero in Europe is found in a Spanish 10thcentury manuscript. In the next few centuries, it spread successfully throughout Christian Europe, despite several official diktats that discouraged or prohibited Indian numerals. In the Chinese empire, the spread of zero started in the 7th century ( $T$ 'ang dynasty), primarily driven through translations of Indian mathematical, astronomical, and Buddhist texts (Martzloff, 1995). In Southeast Asia, imbued with Buddhist cultural influence from India, the use of a circle to denote zero is found in 7th-century inscriptions in Sumatra and Cambodia (Joseph, 1990). In sum, the concept for zero spread successfully from its place of origin to a large part of the Old World in a relatively short span of time.

The emergence of zero as a numerical concept in Jain mathematics was possible because it could free ride on cosmological and philosophical concepts. However, this does not explain its subsequent spread to other cultures. A possible explanation for this success is its minimal violation of intuitive expectations of the number module. Although the number module may be unable to represent zero, zero can become part of its actual domain because the absence of magnitude can be easily mapped onto the absence of stuff in the world. This counterintuitiveness may give zero a memory advantage, as illustrated in a series of experiments that probed the understanding of zero in young children (Wellman \& Miller, 1986). As expected, the development of the zero concept is different from that of other natural numbers. Young preschoolers simply treat zero as synonymous for nothing; they do not realize that it is a numerical concept as well. In magnitude comparison tasks, they are just as likely to say that 0 is larger than 3 as vice versa. By the end of the preschool years, however, most children understand that zero is a numerical concept and correctly identify it as the smallest natural number. Interestingly, the initial difficulties seem to facilitate an understanding of abstract operational rules for zero compared with other numbers. When first-grade students are confronted with an abstract addition or subtraction task, their success is significantly higher for tasks involving 0 than for those involving other small numbers, such as 1 or 2 . For instance, they judge correctly that $a+0=a$ but are at a loss when judging whether $a+2=a$ is false or true. Hence, an early understanding of algebraic rules for zero seems advanced in comparison with other small numbers. The reason for this advantage is clear from the children's justifications for their answers: most state that zero is a special number with special properties. Thus, zero as a numerical concept was long in the making because it is counterintuitive. However, once originated, it became appealing precisely because it violates ontological expectations.

\subsection{Negative numbers}

At first sight, negatives may seem counterintuitive because they violate ontological expectations. However, compared with zero, which is minimally counterintuitive, negative 
numbers provide many violations that ultimately undermine our intuitions about number. Why should a negative times a negative yield a positive? What is the relationship between the value of -3 and its absolute value $|3|$ ? From an evolutionary point of view, it is unlikely that the mental number line would be capable of handling negative numbers because it is empirically impossible to experience negative numerosities. In one number comparison task (Fischer, 2003), adults were presented with pairs of positive integers, a positive and a negative integer, or negative integers. The subjects showed faster response times for small absolute numerosities (e.g., -3, 2) than for larger ones (e.g., -4, 9). However, response times were especially slow in trials with two negative numbers, suggesting that our mental number line does not extend to negative integers. Another line of evidence for the nonintuitiveness of negatives comes from education: eighth-grade students were probed on their knowledge of the minus sign in solving first-grade equations (Vlassis, 2004). Despite their prior knowledge of algebra and negative integers, error rates soared when they were confronted with negatives in equations, especially when compared with equations that involved only positive integers. Interviews with these pupils revealed that they primarily relied on procedural rules learnt by heart. Even high-level students experienced difficulties explaining these procedures, showing that their performance is due to a studious application of rules rather than an intrinsic understanding of negative numerosities. In their answers, they seldom appealed to the mental number line or other models such as explaining negatives in terms of gains and debts. Because negative numbers are nonintuitive, we may ask whether they are still part of the actual domain of the number module. As Fischer (2003) demonstrated, negative integers show similar distance and size effects as do positive integers, although they are processed markedly slower. Thus, intuitions provided by the number module still structure this concept. This can be seen in many other types of nonintuitive thought. For instance, when subjects are asked to invent extraterrestrial life forms, they do not produce a limitless variety of beings but rather draw upon their knowledge of terrestrial animals to structure their imagined beings (e.g., bilateral symmetry, sensory organs), as do professional science fiction writers (Ward, 1994). Likewise, negative numbers are structured by analogy with the positives: they are represented on a mental number line that runs backward.

In contrast to zero, which was readily assimilated as soon as it reached Western mathematicians, negative numbers were poorly received. Most Western 16th- and 17thcentury mathematicians, including Pascal and Vieta, rejected or questioned negative numbers (Buzaglo, 2002). As late as 1830, the eminent mathematician De Morgan wrote that " $3-8$ is an impossibility; it requires you to take from 3 more than there is in 3, which is absurd" (cited in Greer, 2004, p. 541). The struggle with the negative integers' ontological status may have contributed to their poor cultural transmission and long development in Western mathematics. Indeed, in the history of science, we can find numerous examples of how intuitive notions, based on the proper domains of various modules, have restrained and slowed down scientific progress. For example, in biology, the widely held belief that species have unchanging essences is deeply incompatible with evolutionary theories which hold that species can evolve into new species. As Hull (1964) put it, essentialism in taxonomy (and biology in general) resulted in 2000 years of stasis; before Darwin and other evolutionary theorists, biologists such as Linnaeus (1707-1778) did not make any significant theoretical contribution to taxonomic theory since Aristotle (384-322 BC) laid its foundations. Likewise, not until the 
late 19th century did mathematicians finally reject the long-held doctrine that negative numbers are impossible (Greer, 2004). Arabian mathematicians rejected negatives altogether. Indeed the term algebra, derived from the seminal text by al-Khwarizmi, Al-jabr wa'l muqaal-jabr (830), means restoration, as in the sense of 'adding equal terms to both sides of an equation to remove negative quantities' (Stedall, 2001). Although Chinese mathematicians did use negative integers to solve equations, they were reluctant to accept a negative as a result of an equation, resulting in many problematic reformulations of what would otherwise have been straightforward solutions (Joseph, 1990). Initially, only medieval Indian mathematicians developed a system to deal with negative quantities. Prior to the 19th century, European mathematicians such as Leibniz who accepted negatives did so despite their apparent clash with numerical intuitions, emphatically stressing their usefulness in calculations. Like other nonintuitive concepts, their full acceptance requires a highly institutionalized context. Only with the advent of 19th-century mathematical institutions and formalization as part of the curriculum of the new research-oriented universities did negatives become incorporated in Western mathematics (Restivo, 1992). One of the reasons why European mathematicians successfully integrated so many nonintuitive concepts (e.g., imaginary numbers, infinite sets) may be that they consistently strove to externalize such concepts in a consistent set of symbols. This cognitive process, referred to as active externalism (Clark \& Chalmers, 1998), is the process by which computations performed in the mind are extended into the world. By assigning a specific symbol (e.g., the minus sign) to nonintuitive ideas, they become easier to handle and to remember. This process of externalism protects nonintuitive concepts from the corrosive effects of biased cultural transmission. Once mathematical concepts are nested outside the brain, their evolution and cultural transmission are less vulnerable to corruption by individual mathematicians or to competition from ideas that are easier to learn, that speak more to the imagination, or that pose less computational demands. They gain a degree of autonomy that would be impossible to attain were they represented in the mind alone. This externalization is sustained by the highly institutionalized context of research programs in universities, scientific journals, and mathematical societies.

In sum, although negative numbers are still part of the actual domain of the number module, they are nonintuitive and therefore difficult to transmit and reconstruct. As they do not activate an evolved inference system and therefore are supported by few inductive inferences, concepts as these require a long training period and external storage of information to overcome cognitive limitations. Indeed, the history of Western mathematics from the 19th century onward is characterized by an increased acceptance of nonintuitive concepts, including negative numbers, which goes hand in hand with an increased institutionalization of mathematical practice.

\section{Conclusion}

In this study, I examined the cultural transmission of numerical concepts through an epidemiological approach. This research on the history of number concepts provides evidence 
for continuity between science and cognitive evolution. Historical evidence indicates that the emergence and subsequent spread of cultural number concepts are influenced by the evolved structure of the human brain. The multiple and frequent cultural invention of positive integers can be explained as a result of their close fit with intuitions provided by the number module. The anatomical proximity of this module to other modules involved in counting by the sequential tagging procedure further adds to their salience. By being rooted in more than one conceptual module, including body-part recognition and linguistic skills, positive integers are easy to learn and to transmit. The unique invention of zero as a numerical concept and its successful spread can be seen as a result of content-biased cultural transmission. Its counterintuitiveness facilitated its cultural transmission; once emerged, it could easily spread to neighboring cultures with positional systems. In contrast, negative numbers have spread marginally and late from their place of origin due to their nonintuitiveness. Although they are part of the actual domain of the number module, they cannot be grasped intuitively and therefore need a highly institutionalized context to thrive. Active externalism allows for the storage and representation of such nonintuitive information, thereby making it less vulnerable to the corrosive effects of content-biased cultural transmission. These results can shed light on the question of to what extent evolved cognitive capacities that are universal in Homo sapiens are sufficient to promote scientific thought. In human culture, the proper and actual domains of any conceptual module rarely overlap entirely. In some cultural domains of expertise, this overlap is at times so marginal that cultural transmission can only take place within a highly institutionalized context, characterized by active externalism and dedicated highly trained personnel. Without these, humans would perhaps only be able to transmit intuitive and minimally counterintuitive concepts. To date, the epidemiological approach has been applied to biology and religion. This examination of the epidemiology of number representations opens the possibility that this approach can be applied to the study of other scientific disciplines for which evidence exists that they are also governed by evolved conceptual modules, such as physics and psychology.

\section{Acknowledgments}

I gratefully acknowledge all the useful comments on an earlier draft, especially those by Jean Paul Van Bendegem, M. Afzal Upal and Johan De Smedt. I also wish to thank three anonymous reviewers for their helpful suggestions.

\section{References}

Alibali, M. W., \& DiRusso, A. A. (1999). The function of gesture in learning to count: More than keeping track. Cognitive Development, 14, 37-56.

Antell, S. E., \& Keating, D. P. (1983). Perception of numerical invariance in neonates. Child Development, 54, $697-701$. 
Atran, S. (1998). Folk biology and the anthropology of science: Cognitive universals and cultural particulars. Behavioral and Brain Sciences, 21, 547-569.

Barrett, J. L. (1998). Cognitive constraints on Hindu concepts of the divine. Journal for the Scientific Study of Religion, 37, 608-619.

Barrett, J. L., \& Keil, F. C. (1996). Conceptualizing a nonnatural entity: Anthropomorphism in god concepts. Cognitive Psychology, 31, 219-247.

Boyer, P. (2000). Evolutionary psychology and cultural transmission. American Behavioral Scientist, 43, 987-1000.

Boyer, P. (2001). Religion explained. The evolutionary origins of religious thought. New York: Basic Books.

Boyer, P., \& Ramble, C. (2001). Cognitive templates for religious concepts: Cross-cultural evidence for recall of counter-intuitive representations. Cognitive Science, 25, 535-564.

Brewer, W. F., Chinn, C. A., \& Samarapungavan, A. (2000). Explanation in scientists and children. In F. C. Keil, \& R. A. Wilson (Eds.), Explanation and cognition (pp. 279-298). Cambridge: MIT Press.

Butterworth, B. (1999). The mathematical brain. London: Macmillan.

Buzaglo, M. (2002). The logic of concept expansion. Cambridge: Cambridge University Press.

Callaghan, T., Rochat, P., Lillard, A., Claux, M. L., Odden, H., Itakura, S., Tapanya, S., \& Singh, S. (2005). Synchrony in the onset of mental-state reasoning: Evidence from five cultures. Psychological Science, 16, 378-384.

Caramazza, A., \& Mahon, B. Z. (2003). The organization of conceptual knowledge: The evidence from categoryspecific deficits. Trends in Cognitive Sciences, 7, 354-361.

Clark, A., \& Chalmers, D. (1998). The extended mind. Analysis, 58, 7-19.

Cordes, S., Gelman, R., \& Gallistel, C. R. (2001). Variability signatures distinguish verbal from nonverbal counting for both large and small numbers. Psychonomic Bulletin \& Review, 8, 698-707.

Cosmides, L., \& Tooby, J. (1994). Origins of domain specificity: The evolution of functional organization. In L. A. Hirschfeld, \& S. A. Gelman (Eds.), Mapping the mind. Domain specificity in cognition and culture (pp. 85-116). Cambridge: Cambridge University Press.

Dehaene, S. (1997). The number sense. New York: Oxford University Press.

Dehaene, S., Spelke, E., Pinel, P., Stanescu, R., \& Tsivkin, S. (1999). Sources of mathematical thinking: Behavioral and brain-imaging evidence. Science, 284, 970-974.

d'Errico, F. (1998). Palaeolithic origins of artificial memory systems: An evolutionary perspective. In C. Renfrew, \& C. Scarre (Eds.), Cognition and material culture: The archaeology of symbolic storage (pp. 19-50). Cambridge: McDonald Institute for Archaeological Research.

Eger, E., Sterzer, P., Russ, M. O., Giraud, A. -L., \& Kleinschmidt, A. (2003). A supramodal number representation in human intraparietal cortex. Neuron, 37, 1-20.

Fischer, M. H. (2003). Cognitive representation of negative numbers. Psychological Science, 14, 278-282.

Gallistel, C. R. (1990). The organization of learning. Cambridge, MA: MIT Press.

Gallistel, C. R. (1995). The replacement of general-purpose theories with adaptive specializations. In M. S. Gazzaniga (Ed.), The cognitive neurosciences (pp. 1255-1267). Cambridge: MIT Press.

Gallistel, C. R., \& Gelman, R. (2000). Non-verbal numerical cognition: From reals to integers. Trends in Cognitive Sciences, 4, 59-65.

Geary, D. C. (1995). Reflections of evolution and culture in children's cognition. Implications for mathematical development and instruction. American Psychologist, 50, 24-37.

Goldin-Meadow, S., \& Wagner, S. M. (2005). How our hands help us learn. Trends in Cognitive Sciences, 9, $234-241$.

Gordon, P. (2004). Numerical cognition without words: Evidence from Amazonia. Science, 306, 496-499.

Greer, B. (2004). The growth of mathematics through conceptual restructuring. Learning and Instruction, 14, $541-548$.

Hauser, M. D. (2005). Our chimpanzee mind. Nature, 437, 60-63.

Hauser, M. D., Carey, S., \& Hauser, L. B. (2000). Spontaneous number representation in semi-free-ranging rhesus monkeys. Proceedings of the Royal Society London B, 267, 829-833.

Hull, D. L. (1964). The effect of essentialism on taxonomy - two thousand years of stasis (I). British Journal for the Philosophy of Science, 15, 314-326. 
Ifrah, G. (1985). Les chiffres, ou l'histoire d'une grande invention. Paris: Laffont.

Joseph, G. G. (1990). The crest of the peacock. Non-European roots of mathematics. London: Penguin.

Le Clec'H, G., Dehaene, S., Cohen, C., Mehler, J., Dupoux, E., Poline, J. B., Lehéricy, S., van de Moortele, P. F., $\&$ Le Bihan, D. (2000). Distinct cortical areas for names of numbers and body parts independent of language and input modality. NeuroImage, 12, 381-391.

Martzloff, J. -C. (1995). Review of Lam Lay Yong and Ang Tian Se (1992). Fleeting footsteps: Tracing the conception of arithmetic and algebra in ancient China. Historia Mathematica, 22, 67-87.

McCauley, R. N. (2000). The naturalness of religion and the unnaturalness of science. In F. C. Keil, \& R. A. Wilson (Eds.), Explanation and cognition (pp. 61-85). Cambridge: MIT Press.

McComb, K., Packer, C., \& Pusey, A. (1994). Roaring and numerical assessment in contests between groups of female lions, Panthera leo. Animal Behaviour, 47, 379-387.

Moyer, R. S., \& Landauer, T. K. (1967). Time required for judgements of numerical inequality. Nature, 215, $1519-1520$.

Nieder, A., \& Miller, E. K. (2003). Coding of cognitive magnitude: Compressed scaling of numerical information in the primate prefrontal cortex. Neuron, $37,149-157$.

Pica, P., Lemer, C., Izard, V., \& Dehaene, S. (2004). Exact and approximate arithmetic in an Amazonian indigene group. Science, 306, 499-503.

Restivo, S. (1992). Mathematics in society and history: Sociological inquiries. Dordrecht: Kluwer Academic Publishers.

Sandrini, M., Rossini, P. M., \& Miniussi, C. (2004). The differential involvement of inferior parietal lobule in number comparison: A rTMS study. Neuropsychologia, 42, 1902-1909.

Sarnecka, B. W., \& Gelman, S. A. (2004). Six does not just mean a lot: Preschoolers see number words as specific. Cognition, 92, 329-352.

Saxe, G. B. (1981). Body parts as numerals: A developmental analysis of numeration among the Oksapmin in Papua New Guinea. Child Development, 52, 306-316.

Simon, O., Mangin, J. -F., Cohen, L., Le Bihan, D., \& Dehaene, S. (2002). Topographical layout of hand, eye, calculation, and language-related areas in the human parietal lobe. Neuron, 33, 475-487.

Sperber, D. (1994). The modularity of thought and the epidemiology of representations. In L. A. Hirschfeld, \& S. A. Gelman (Eds.), Mapping the mind. Domain specificity in cognition and culture (pp. 39-67). Cambridge: Cambridge University Press.

Sperber, D. (1996). Explaining culture. A naturalistic approach. Oxford: Blackwell.

Sperber, D., \& Hirschfeld, L. A. (2004). The cognitive foundations of cultural stability and diversity. Trends in Cognitive Sciences, 8, 40-46.

Stedall, J. A. (2001). Of our own nation: John Wallis's account of mathematical learning in medieval England. Historia Mathematica, 2, 73-122.

Temple, E., \& Posner, M. I. (1998). Brain mechanisms of quantity are similar in 5-year-old children and adults. Proceedings of the National Academy of Sciences of the United States of America, 95, 7836-7841.

Tooby, J., Cosmides, L. (1995). Foreword. In S. Baron-Cohen (Ed.), Mindblindness. An essay on autism and theory of mind (pp.xi-xviii). Cambridge: MIT Press.

Uller, C., Hauser, M., \& Carey, S. (2001). Spontaneous representation of number in cotton-top tamarins (Saguinus oedipus). Journal of Comparative Psychology, 115, 248-257.

Uller, C., Jaeger, R., Guidry, G., \& Martin, C. (2003). Salamanders (Plethodon cinereus) go for more: Rudiments of number in an amphibian. Animal Cognition, 6, 105-112.

Varley, R. A., Klessinger, N. J. C., Romanowski, C. A. J., \& Siegal, M. (2005). Agrammatic but numerate. Proceedings of the National Academy of Sciences of the United States of America, 102, 3519-3524.

Vlassis, J. (2004). Making sense of the minus sign or becoming flexible in 'negativity'. Learning and Instruction, $14,469-484$.

Ward, T. B. (1994). Structured imagination: The role of category structure in exemplar generation. Cognitive Psychology, 27, 1-40.

Wellman, H. M., \& Miller, K. F. (1986). Thinking about nothing: Development of concepts of zero. British Journal of Developmental Psychology, 4, 31-42. 
Wynn, K. (1992). Addition and subtraction by human infants. Nature, 358, 749-750.

Wynn, K. (1998). Psychological foundations of number: Numerical competence in human infants. Trends in Cognitive Sciences, 2, 296-303.

Wynn, K., \& Chiang, W. (1998). Limits to infants' knowledge of objects: The case of magical appearance. Psychological Science, 9, 448-455.

Xu, F., \& Spelke, E. S. (2000). Large number discrimination in 6-month-old infants. Cognition, 74, B1-B11. 